EPJ Web of Conferences 113,04017 (2016)

DOI: $10.1051 /$ epjconf/201611304017

(C) Owned by the authors, published by EDP Sciences, 2016

\title{
Deuteron form factor measurements at low momentum transfers
}

B. S. Schlimme ${ }^{1, a}$, P. Achenbach ${ }^{1}$, J. Beričič², R. Böhm ${ }^{1}$, D. Bosnar ${ }^{3}$, L. Correa ${ }^{4}$, M. O. Distler ${ }^{1}$, A. Esser ${ }^{1}$, H. Fonvieille ${ }^{4}$, I. Friščić ${ }^{3}$, K. A. Griffioen ${ }^{5}$, Y. Huan ${ }^{5}$, S. Kegel ${ }^{1}$, Y. Kohl ${ }^{1}$, H. Merkel ${ }^{1}$, M. Mihovilovič ${ }^{1}$, J. Müller ${ }^{1}$, U. Müller ${ }^{1}$, J. Pochodzalla ${ }^{1}$, M. Schoth ${ }^{1}$, F. Schulz ${ }^{1}$, C. Sfienti ${ }^{1}$, S. Širca ${ }^{2,6}$, S. Štajner ${ }^{2}$, M. Thiel ${ }^{1}$, and A. Weber ${ }^{1}$

${ }^{1}$ Institut für Kernphysik, Johannes Gutenberg-Universität Mainz, D-55099 Mainz, Germany

${ }^{2}$ Jožef Stefan Institute, SI-1000 Ljubljana, Slovenia

${ }^{3}$ Department of Physics, University of Zagreb, HR-10002 Zagreb, Croatia

${ }^{4}$ Clermont Université, Université Blaise Pascal, F-63000 Clermont-Ferrand, France

${ }^{5}$ College of William and Mary, Williamsburg, VA 23187, USA

${ }^{6}$ Department of Physics, University of Ljubljana, SI-1000 Ljubljana, Slovenia

\begin{abstract}
A precise measurement of the elastic electron-deuteron scattering cross section at four-momentum transfers of $0.24 \mathrm{fm}^{-1} \leq Q \leq 2.7 \mathrm{fm}^{-1}$ has been performed at the Mainz Microtron. In this paper we describe the utilized experimental setup and the necessary analysis procedure to precisely determine the deuteron charge form factor from these data. Finally, the deuteron charge radius $r_{d}$ can be extracted from an extrapolation of that form factor to $Q^{2}=0$.
\end{abstract}

\section{Introduction}

Electromagnetic form factors of light nuclei can be measured in elastic electron scattering. The results can be compared to predictions obtained by different theoretical approaches (for instance, see [1]) and therefore offer a unique opportunity to test our understanding of nuclear dynamics. In addition, charge radii can be determined from precise measurements of the charge form factors at low momentum transfers $Q$.

Assuming single photon exchange, the cross section for unpolarized electron-deuteron scattering can be written as

$$
\begin{aligned}
\frac{\mathrm{d} \sigma}{\mathrm{d} \Omega}= & \sigma_{\mathrm{NS}}\left[A\left(Q^{2}\right)+B\left(Q^{2}\right) \tan ^{2} \frac{\theta_{e}}{2}\right], \\
A\left(Q^{2}\right) & =G_{\mathrm{C}}^{2}\left(Q^{2}\right)+\frac{8}{9} \eta^{2} G_{\mathrm{Q}}^{2}\left(Q^{2}\right)+\frac{2}{3} \eta G_{\mathrm{M}}^{2}\left(Q^{2}\right), \\
B\left(Q^{2}\right) & =\frac{4}{3} \eta(1+\eta) G_{\mathrm{M}}^{2}\left(Q^{2}\right),
\end{aligned}
$$

where $\sigma_{\mathrm{NS}}$ is the Mott differential cross section multiplied by the deuteron recoil factor, $\theta_{e}$ the electron scattering angle and $\eta=\frac{Q^{2}}{4 M^{2}}$ with the deuteron mass $M . G_{\mathrm{C}}, G_{\mathrm{Q}}$ and $G_{\mathrm{M}}$ are the the charge, quadrupole

a e-mail: schlimme@kph.uni-mainz.de 
and magnetic form factors, respectively. In the kinematic regime investigated within the experiment, the cross section is dominated by the contribution of the deuteron charge form factor $G_{\mathrm{C}}\left(Q^{2}\right)$. Taking into account corrections due to the small contributions of the quadrupole and the magnetic form factors, the cross section measurements allow for a determination of the charge form factor as a function of the momentum transfer. From the extrapolation of $G_{\mathrm{C}}\left(Q^{2}\right)$ to $Q^{2}=0$, the deuteron radius can then be extracted from the slope $\mathrm{d} G_{\mathrm{C}} / \mathrm{d} Q^{2}$.

The interest in the deuteron radius is renewed in the context of the proton radius puzzle, pointing out the inconsistency between proton radius values obtained using different experimental techniques: The recommended deuteron radius value from the CODATA group is dominated by the determined value for the proton radius combined with isotope shift data, relating the proton and deuteron radii. Including the result from muonic hydrogen measurements [2] results in a drastic change of $r_{d}$. An overview of deuteron charge radius results is illustrated in Fig. 1.

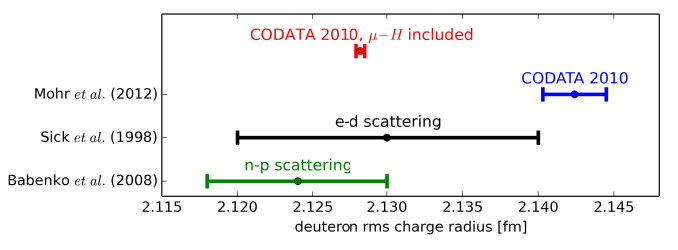

Figure 1. Various determinations of the deuteron charge radius $r_{d}$ [3-5]. The uncertainty of the radius determination from electron-deuteron scattering is too large to reasonably distinguish between the two quoted CODATA values with and without inclusion of the muonic hydrogen result.

\section{Experiment}

Deuteron form factor measurements have been carried out by the A1 Collaboration at the spectrometer facility [6] at MAMI [7] to considerably improve the current form factor data base and to provide an extraction of the deuteron radius with a significant smaller uncertainty. Data were taken in 2014 during three weeks of beam time. Cross section measurements of the elastic $d\left(e, e^{\prime}\right) d$ reaction have been performed for three different beam energies $\left(E_{e}=180,315\right.$, and $\left.450 \mathrm{MeV}\right)$. The scattered electrons were detected with three high resolution magnetic spectrometers simultaneously (spectrometers $\mathrm{A}, \mathrm{B}$, and C) to maximize the covered angular range and the internal redundancy in the data. The spectrometers have a relative momentum resolution of $\sim 10^{-4}$ and an angular resolution at the target of $\sim 3 \mathrm{mrad}$ [6]. The angular acceptance of the spectrometers is well defined by collimators. The central electron scattering angles were varied in the range between $15^{\circ}$ and $107^{\circ}$ by changing the positions of the spectrometers. The chosen kinematic settings reach down in $Q^{2}$ as far as possible with the available setup (down to $Q=0.24 \mathrm{fm}^{-1}$, similar to lowest published data), and have high redundancy in the region $0.5 \mathrm{fm}^{-1} \leq Q \leq 1.2 \mathrm{fm}^{-1}$ where sensitivity to the rms-radius is high [1]. Altogether, almost 200 different kinematic settings have been investigated during the beam time.

The electron beam impinged on a liquid deuterium target with beam currents between $\sim 1 \mathrm{nA}$ and $4 \mu \mathrm{A}$, which were measured with Foerster probes and a pA-meter. Vertical drift chambers (VDCs) were used for tracking, scintillation detectors for trigger and timing purposes, and a threshold-gasCherenkov detector for electron identification in the offline analysis. Due to the large cross sections, the low background, and the relatively high data taking rate of about $500 \mathrm{~Hz}$ per spectrometer, all kinematic settings could be measured with reasonable statistics during the available time. Hence, the statistical errors of the resulting cross section determinations are well below the $1 \%$ level for most of the settings. Usually only one spectrometer angle was varied at a time, allowing to monitor changes in the luminosity with the other two spectrometers. The beam delivery was generally not stopped during angle changes, feasible through the use of a remote spectrometer movement control, to minimize possible beam parameter changes. 


\section{Data analysis}

For each recorded event the raw data of the individual detectors are considered to reconstruct kinematic quantities like the angle and the momentum of the detected particle and the reaction vertex. The VDC and scintillator data is used for track reconstruction of the detected particles in the focal plane of the spectrometers. The well known optics are used to reconstruct the particle tracks at the target position. Identification of elastic electron-deuteron scattering events is then accomplished via comparison of the detected energy $E_{\exp }^{\prime}$ with the energy calculated from the detected scattering angle, $E^{\prime}\left(\theta_{\exp }\right)$, see Fig. 2. Comparison of the experimental spectra with the spectra from a simulation then yields the

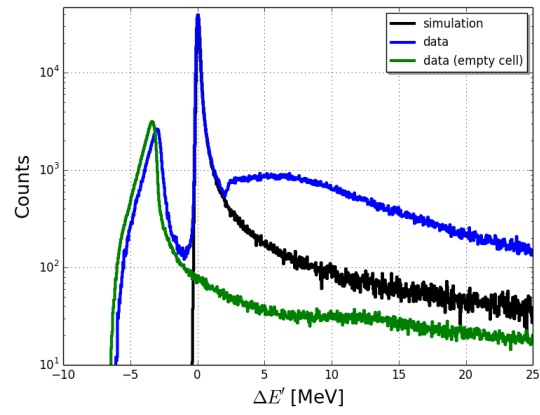

Figure 2. Identification of elastic electron-deuteron scattering events. Data (blue) and simulation (black) for spectrometer A at $\theta_{e}=23.6^{\circ}$ and $E_{e}=315 \mathrm{MeV}$. The additional large contribution for $\Delta E^{\prime}=E^{\prime}\left(\theta_{\exp }\right)-E_{\exp }^{\prime}>2.2 \mathrm{MeV}$ from the deuteron breakup reaction can be clearly separated from the elastic peak, which is located around zero. Furthermore, there is a contribution from electrons originating from the foils surrounding the deuterium target. Empty cell data, taken for the purpose of background subtraction, are shown in green.

experimental cross sections relative to the cross sections employed in the simulation. The simulation accounts for leading radiative processes beyond the one photon exchange approximation including the generation of the radiative tail, energy loss and straggling of the electrons in the target and window materials. Not all of the necessary corrections have been applied, yet. For instance, there is a contribution from background electrons originating from the target foils beneath the elastic deuteron peak. That contribution is in general larger for smaller angles. Since the deuteron charge radius will be determined from the slope of the extracted charge form factor with respect to the momentum transfer, a careful study of this kind of systematic effects is mandatory. Empty cell data have been taken for the purpose of background subtraction. Due to a slightly different energy loss situation when measuring on an empty cell without deuterium gas inside, there is a slight mismatch between the empty cell spectrum and the spectrum measured with the deuterium target which needs to be accounted for. To avoid an erroneous background subtraction, a simulation of the target wall contribution, including the contribution from excited states, is being developed to study that effect in detail. To illustrate the statistical quality of the data, a preliminary cross section result for one particular data set is shown in Fig. 3.

\section{Outlook}

The extraction of the individual cross section values is clearly the central part of the analysis. Several crucial corrections have not been applied yet, for instance Coulomb corrections or the mentioned background subtraction. Also, the modelling of the spectrometer resolutions, needed for a precise comparison between data and simulation, has to be finalized. Moreover, the determination of the luminosity is actually solely based on the measured target parameters and the beam current, and can be improved by the comparison of the count rates of the spectrometers, which were not moved between a setting change of the third spectrometer. Once all the necessary corrections and crosschecks have been performed, the obtained cross sections can be used to extract the charge form factor of the deuteron, $G_{\mathrm{C}}^{2}\left(Q^{2}\right)$. An appropriate fitting procedure will be applied for this purpose. From 


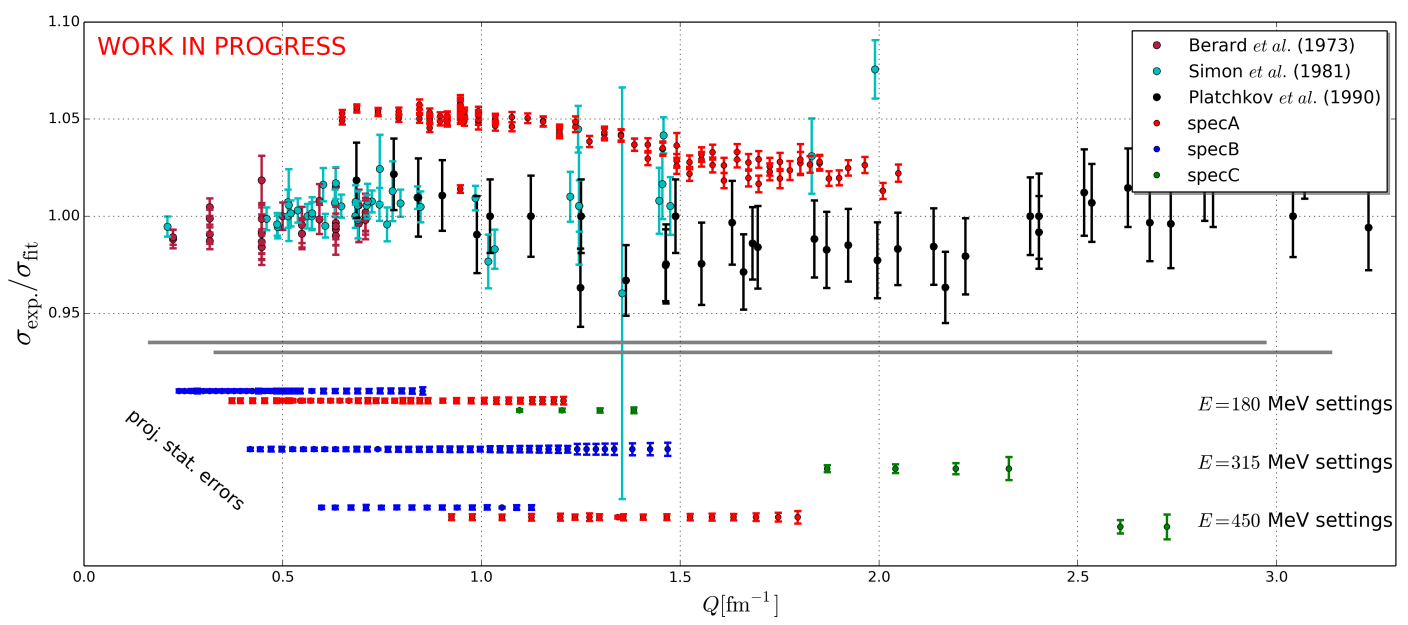

Figure 3. Top: Experimental cross sections divided by the cross sections calculated with the form factor parametrization of [8] (Sum-of-Gaussians) for one particular data set (red circles) at $E_{e}=315 \mathrm{MeV}$ (statistical errors only). The reevaluated (see [4]) most precise existing low- $Q$ data sets [9-11] are shown for comparison. The beam current, which enters the luminosity determination, was measured with a pA-meter, which has not been calibrated at this stage of the analysis. This can result in a systematic shift of our data compared to the previous data, as can be observed in the figure. Finally, the absolute normalization will be fixed by the known values of the form factors at $Q^{2}=0$. There is an additional rise of our data at smaller momentum transfers $Q$ essentially caused by background produced at the target foils, which has not been subtracted, yet. Bottom: Projected statistical errors of the other data sets of this experiment.

the slope of $G_{\mathrm{C}}^{2}\left(Q^{2}\right)$ at $Q^{2}=0$, the deuteron radius can then be determined, with the aim to provide additional insight to the proton radius puzzle.

\section{Acknowledgements}

This work was supported by the Deutsche Forschungsgemeinschaft with the Collaborative Research Center 1044: The Low-Energy Frontier of the Standard Model. From Quarks and Gluons to Hadrons and Nuclei.

\section{References}

[1] L.E. Marcucci et al., arXiv:1504.05063 [nucl-th]

[2] R. Pohl et al., Nature 466, 213 (2010)

[3] P.J. Mohr et al., Rev. Mod. Phys. 84, 1527 (2012)

[4] I. Sick and D. Trautmann, Nucl. Phys. A 637, 559 (1998)

[5] V.A. Babenko and N.M. Petrov, Phys. Atom. Nucl. 71, 1730 (2008)

[6] K.I. Blomqvist et al., Nucl. Instrum. Meth. A 403, 263 (1998)

[7] H. Herminghaus et al., Nucl. Instrum. Meth. 138, 1 (1976)

[8] D. Abbott et al., Eur. Phys. J. A 7 (2000) 421

[9] R.W. Berard et al., Phys. Lett. B 47, 355 (1973)

[10] G.G. Simon et al., Nucl. Phys. A 364, 285 (1981)

[11] S. Platchkov et al., Nucl. Phys. A 510, 740 (1990) 